\title{
The Comparison of Outcomes between Video-assisted Thoracscopic and Open Surgery for Esophageal Cancer
}

\author{
Hiroshi Tamagawa' ${ }^{1)}$, Masakatsu Numata ${ }^{1)}$, Toru Aoyama ${ }^{1)}$, Ayako Tamagawa $^{1)}$, Keisuke Komori $^{2)}$, \\ Yukio Maezawa $^{1)}$, Kazuki Kano ${ }^{1)}$, Keisuke Kazama ${ }^{1)}$, Masaaki Murakawa ${ }^{1)}$, Yosuke Atsumi ${ }^{1)}$, \\ Kentaro Hara $^{1)}$, Shinnosuke Kawahara ${ }^{1)}$, Takanobu Yamada ${ }^{2)}$, Takashi Ogata'), Takashi Ohshima ${ }^{2)}$, \\ Norio Yukawa ${ }^{1)}$, Munetaka Masuda ${ }^{1)}$, and Yasushi Rino ${ }^{1)}$ \\ ${ }^{1)}$ Department of Surgery, Yokohama City University \\ ${ }^{2)}$ Department of Gastrointestinal Surgery, Kanagawa Cancer Center
}

\begin{abstract}
Background: Minimally invasive esophagectomy theoretically has advantages over open esophagectomy. The purpose of the present study was to compare the short- and long-term outcomes of patients who underwent video-assisted thoracoscopic esophagectomy (VATE) or conventional open esophagectomy (OE) for esophageal carcinoma.

Methods: A total of 122 patients who underwent radical esophagectomy via VATE (VATE group, $\mathrm{N}=87$ ) and OE (OE group, $\mathrm{N}=35$ ) for esophageal carcinoma between 2005 and 2018 were retrospectively enrolled in this study, and the postoperative outcomes were compared.

Results: The OE group had younger patients and more patients who received neo-adjuvant therapy that the VATE group. The procedure time in the OE group was also shorter than that in the VATE group $(8.22 \mathrm{vs} .10 .28 \mathrm{~h}, \mathrm{P}<0.001)$. Intraoperative blood loss was similar between the groups $(\mathrm{P}=0.775)$. There were no significant differences in the total number of dissected lymph nodes between the groups (OE: 33, VATE: 37, $\mathrm{P}=0.482$ ). The incidence of severe complications was lower in the VATE group than in the OE group $(44.8 \%$ vs. $65.7 \%, \mathrm{P}=0.037)$. With a median follow-up of 26 months, the 3-year overall survival and disease-free survival were similar between the two groups.

Conclusion: VATE for esophageal carcinoma is associated with more favorable short-term outcomes and equal oncological outcomes compared with OE.
\end{abstract}

Keywords: Esophageal cancer; Video-assisted thoracoscopic surgery; outcomes; Open surgery

(Received July 28, 2020; Accepted September 10, 2020)

\section{Introduction}

Esophageal cancer is the eighth-most common type of cancer and the sixth-most common cause of death due to cancer in the world ${ }^{1}$. More than 456,000 new esophageal cancer diagnoses and 400,000 deaths per year were reported to occur worldwide in $2012^{1)}$. The combination of neoadjuvant chemoradiotherapy and complete surgical resection has become the standard treatment of advanced esophageal cancer ${ }^{2}$. However, although the resection rate has increased gradually, patients with esophageal cancer often develop tumor recurrence. The prognosis for esophageal cancer patients after curative resection remains poor with a 3- and 5-year overall survival (OS) of $44 \%$ and $26 \%$, respectively ${ }^{3)}$. Furthermore, radical

Correspondence to: Hiroshi Tamagawa, Department of Surgery, Yokohama City University, 3-9 Fukuura, Kanazawa-ku, Yokohama 236-0004, Japan. E-mail: rinta77nosuke@amber.plala.or.jp esophagectomy is a major undertaking and is associated with significant morbidity and mortality, especially in elderly patients ${ }^{4)}$. Therefore, it is important to control the postoperative complications.

Video-assisted thoracoscopic esophagectomy (VATE) is an established minimally invasive procedure that was first reported in 1992 by Cuschieri ${ }^{5)}$. VATE has been reported to improve the short-term outcomes in terms of less blood loss and analgesic requirements, quicker recovery and a shorter hospital stay ${ }^{6}$. However, some reports have shown various results in the comparison of the short-term results between VATE and conventional open surgery $(\mathrm{COS})^{7-10)}$, and the survival outcomes of VATE for esophageal carcinoma are still unclear.

The present study therefore compared the short- and long-term operative outcomes between VATE and COS in patients with esophageal cancer with a retrospective cohort analysis. 


\section{Patients and Methods}

\section{Patient data}

A database with primary esophageal cancer patients who underwent surgical treatment at Yokohama City University from January 2005 to September 2018. The patients who had undergone palliative or non-curative surgery were excluded. All patients were pathologically determined to have squamous cell carcinoma of clinical stage IB to III (excluding T4).

\section{Surgical procedure}

Conventional open transthoracic esophagectomy via right thoracotomy or video-assisted surgery with two- or three-field lymphadenectomy was performed, along with reconstruction through the retrosternal route or posterior mediastinal route and cervical or intrathoracic anastomosis. A feeding tube was routinely placed at the stomach or duodenum. A jejunostomy catheter was routinely placed for postoperative enteral nutrition.

\section{Perioperative care}

All of the patients received the same perioperative management. Antibiotics were administered $30 \mathrm{~min}$ before surgical incision and every $3 \mathrm{~h}$ during the operation as well as on postoperative day (POD) 2. On POD 1, enteral nutrition and ambulation training were started. On POD 5, oral intake with water and gelatinous foods was initiated, and solid food was started on POD 10.

\section{Follow-up}

All patients were followed-up for five years after surgical curative resection. The serum SCC, CEA and CA19-9 levels and radiological examination findings by computed tomography were checked at least every three months for five years at outpatient clinics. Disease recurrence was diagnosed based on tumor marker levels and radiographic evidence of a new suspicious low-density mass.

\section{Evaluations and statistical analyses}

The significance of the associations between the operative approach and clinicopathological parameters was determined using the $\chi^{2}$ test or Fisher's exact test. The overall survival (OS) was defined as the period from surgery until death. The recurrence-free survival (RFS) was defined as the period from surgery until recurrence or death. The OS and RFS curves were plotted according to the Kaplan-Meier method, and statistical differences between different groups were compared using the logrank test. $\mathrm{P}$ values of $<0.05$ were considered statistically significant.

Statistical analyses were performed using the statistical software program SPSS, ver. 23.0 (IBM, Chicago,
IL, USA). This study was approved by the Institutional Review Board (IRB) Committee of the Yokohama City University. Informed consent to use clinical data without identifying personal data was obtained in all cases.

\section{Results}

\section{Patient characteristics}

A total of 122 patients underwent esophagectomy for esophageal cancer and preoperatively between October 2005 and September 2018. The patients' ages ranged from 40 to 82 years old (median: 68 years old); 106 were male, and 16 were female. Eighty-seven. patients were classified into the VATE group, and 35 patients were classified into the OS group.

\section{Clinicopathological features}

Table 1 summarizes the patients' background with the VATE group and OS group in the present study. There were significant differences in the distribution of age and presence of neo-adjuvant chemotherapy between the two groups. However, there were no significant differences in the gender, body mass index, site of tumor, lymph node dissection or pathological $\mathrm{T}$ and $\mathrm{N}$ factors.

\section{Short-term outcomes}

The short-term outcomes are shown in Table 2. The operation time was significantly longer in the VATE group than in the OS group (10.28 vs. $8.22 \mathrm{~h}, p<0.001)$, but the findings were similar between the groups with regard to blood loss (542 vs. $500 \mathrm{ml} ; p=0.775)$ and the rate of transfusion ( $31.0 \%$ vs. $25.7 \%$; $p=0.560$ ). More lymph nodes were dissected in the VATE group than in the OS group, but the difference was not statistically significant (37 vs. 33; $p=0.482$ ). The postoperative complication rate was lower in the VATE group than in the OS group, with more cases with a Clavien-Dindo classification of grade III (44.8\% vs. $65.7 \% ; p=0.037)$. In addition, respiratory complications were less prevalent in the VATE group than in the OS group (40.1\% vs. $60.0 \%$; $p=0.047$ ). The postoperative hospital stay duration and duration of intensive-care unit (ICU) stay were similar between the groups. The type of recurrence and median time to first cancer recurrence were also similar between the groups. The mortality rate was $0.8 \%$ in this study. There was not significant difference between two groups.

\section{Survival analyses}

The median duration of follow-up was 27 months for the whole cohort (range: $0-116$ months). The OS and RFS curves are shown in Fig. 1. The OS rate at 3 years after surgery was $69.1 \%$ in the VATE group and $53.8 \%$ in the OS group, which was not significantly different ( $\mathrm{p}=$ $0.161)$. The RFS rate at 3 years after surgery was $52.2 \%$ in the VATE group and $39.4 \%$ in the OS group, which 
Table 1 Comparison of patient background factors between video-assisted surgery group and open surgery group

\begin{tabular}{|c|c|c|c|c|}
\hline Variables & All patients $(\mathrm{n}=122)$ & VATS $(\mathrm{n}=87)$ & Open $(n=35)$ & $p$ value \\
\hline Age (years), median (range) & $66(40-82)$ & $70(40-82)$ & $65(49-78)$ & $\mathbf{0 . 0 2 0}$ \\
\hline $\begin{array}{l}\text { Gender } \\
\text { Female } \\
\text { Male }\end{array}$ & $\begin{array}{c}16(13.1 \%) \\
106(86.9 \%)\end{array}$ & $\begin{array}{l}10(11.5 \%) \\
77(88.5 \%)\end{array}$ & $\begin{array}{c}6(17.1 \%) \\
29(82.9 \%)\end{array}$ & 0.403 \\
\hline Body mass index, median (range) & $21.0(14.5-29.0)$ & $20.9(14.5-29.0)$ & $21.2(16.1-27.6)$ & 0.598 \\
\hline $\begin{array}{l}\text { ASA-PS } \\
1 \\
2,3\end{array}$ & $\begin{array}{c}11(9.0 \%) \\
111(91.0 \%)\end{array}$ & $\begin{array}{c}2(5.7 \%) \\
33(94.3 \%)\end{array}$ & $\begin{array}{c}9(10.3 \%) \\
78(89.7 \%)\end{array}$ & 0.580 \\
\hline $\begin{array}{l}\text { Site of tumor } \\
\text { Upper thoracic } \\
\text { Middle thoracic } \\
\text { Lower thoracic }\end{array}$ & $\begin{array}{l}31(26.3 \%) \\
53(44.9 \%) \\
34(28.8 \%)\end{array}$ & $\begin{array}{l}21(25.3 \%) \\
39(47.0 \%) \\
53(27.7 \%)\end{array}$ & $\begin{array}{l}10(28.6 \%) \\
14(40.0 \%) \\
11(31.4 \%)\end{array}$ & 0.784 \\
\hline $\begin{array}{l}\text { Nodal dissection } \\
\text { Two fields } \\
\text { Three firlds } \\
\end{array}$ & $\begin{array}{l}69(57.0 \%) \\
52(43.0 \%) \\
\end{array}$ & $\begin{array}{l}49(56.3 \%) \\
38(43.7 \%)\end{array}$ & $\begin{array}{l}20(58.8 \%) \\
14(41.2 \%)\end{array}$ & 0.803 \\
\hline $\begin{array}{l}\text { Neo-adjuvant chemotherapy } \\
\text { Abcent } \\
\text { present }\end{array}$ & $\begin{array}{l}51(43.2 \%) \\
67(56.8 \%)\end{array}$ & $\begin{array}{l}45(53.6 \%) \\
39(46.1 \%) \\
\end{array}$ & $\begin{array}{c}6(17.6 \%) \\
28(82.4 \%) \\
\end{array}$ & $<0.001$ \\
\hline $\begin{array}{l}\text { Pathological T factor } \\
\text { pT1-2 } \\
\text { pT3 }\end{array}$ & $\begin{array}{l}63(51.6 \%) \\
59(48.4 \%) \\
\end{array}$ & $\begin{array}{l}49(56.3 \%) \\
38(43.7 \%)\end{array}$ & $\begin{array}{l}14(40.0 \%) \\
21(60.0 \%)\end{array}$ & 0.103 \\
\hline $\begin{array}{l}\text { Pathological } \mathrm{N} \text { factor } \\
\text { Abcent } \\
\text { present }\end{array}$ & $\begin{array}{l}62(50.8 \%) \\
60(49.2 \%)\end{array}$ & $\begin{array}{l}45(51.7 \%) \\
42(48.3 \%)\end{array}$ & $\begin{array}{l}17(48.6 \%) \\
18(51.4 \%)\end{array}$ & 0.753 \\
\hline Follow up period, median (range) & $808(4-3479)$ & $829(39-2909)$ & $732(4-3479)$ & 0.934 \\
\hline
\end{tabular}

ASA-PS American Society of Anesthesiologists physical status

Table 2 Relationship of short-term outcomes between video-assisted surgery group and open surgery group

\begin{tabular}{l|c|c|c}
\hline & VATS $(\mathrm{n}=87)$ & Open $(\mathrm{n}=35)$ & $p$ value \\
\hline Operation time (hour) & $10.28(6.11-15.11)$ & $8.22(3.56-11.43)$ & $<\mathbf{0 . 0 0 1}$ \\
\hline Intraoperative bleeding (g) & $542(70-2992)$ & $500(95-3000)$ & 0.775 \\
\hline Transfusion & $27(31.0 \%)$ & $9(25.7 \%)$ & 0.560 \\
\hline Number of dissected LN & $37(13-118)$ & $33(3-86)$ & 0.482 \\
\hline Complications (grade3-) & $39(44.8 \%)$ & $23(65.7 \%)$ & $\mathbf{0 . 0 3 7}$ \\
\hline Respiratory complications & $35(40.1 \%)$ & $21(60.0 \%)$ & $\mathbf{0 . 0 4 7}$ \\
\hline Anastomous leakage & $31(35.6 \%)$ & $11(31.4 \%)$ & 0.658 \\
\hline Recurrent laryngeal nerve palsy & $23(26.7 \%)$ & $7(20.4 \%)$ & 0.483 \\
\hline ICU stay (day) & $5(0-367)$ & $4(0-373)$ & 0.607 \\
\hline Hospital stay (day) & $31(0-412)$ & $24(4-376)$ & 0.626 \\
\hline Recurrence site & & & \\
Liver & 8 & 2 & 0.526 \\
Lung & 9 & 4 & 0.537 \\
Local & 5 & 10 & 0.278 \\
Lymph node & 21 & 0 & 0.611 \\
Brain & 3 & 1 & 0.266 \\
Bone & 3 & & 0.868 \\
\hline
\end{tabular}

1-a. Recurrence free survival

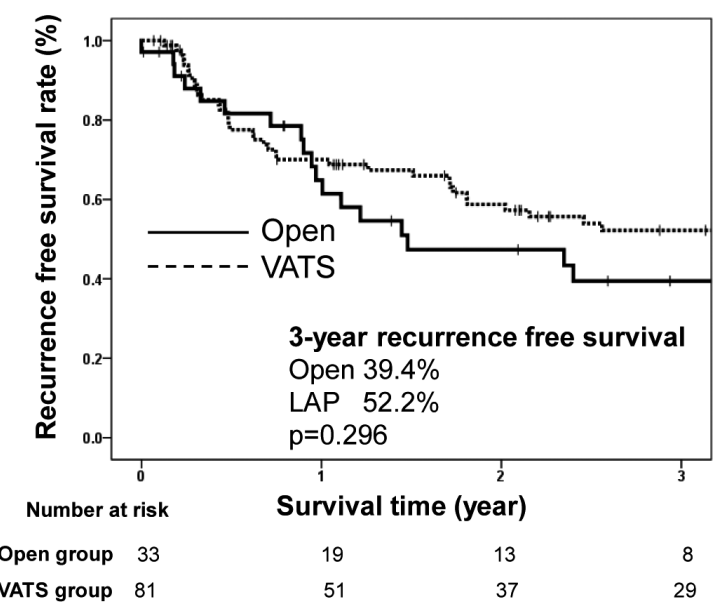

1-b. Overall survival

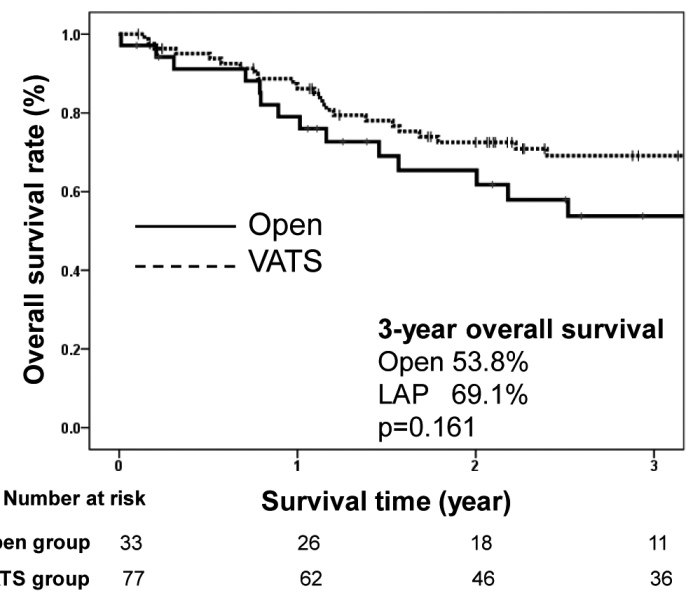

Fig. 1 A comparison of the recurrence-free survival (1-a) and overall survival (1-b) rate in the VATE group and OS group who underwent potentially curative surgery for esophageal cancer. 
was not also significantly different $(\mathrm{p}=0.296)$.

\section{Discussion}

The present study examined whether the approach of operation methods was associated with short- and longterm outcomes in patients who received radical esophagectomy for esophageal cancer. Most of our findings clearly indicated that the site of cancer recurrence, OS and RFS were similar between the two groups during long-term follow-up. In the short-term follow-up, VATE reduced the rate of both postoperative pulmonary complications and severe postoperative complications.

The main concern associated within VATE for esophageal carcinoma is the risk of inadequate tumor resection. However, no marked difference was observed in the rate of margin-free resection or lymph node dissection between VATE and open resection in several comparative studies ${ }^{11,12}$. Furthermore, there are some reports that VATE is superior to OS with regard to the long-term prognosis. Iguchi et al. ${ }^{10)}$ reported that the 5-year OS was significantly better in the VATE group than in the OS group $(69.0 \%$ vs. $35.5 \%$; $p=0.004)$ and a multivariate analysis showed that VATE was a prognostic factor of the OS $(\mathrm{p}<0.001)$ and RFS $(\mathrm{p}=0.032)$ in patients with stage II/III esophageal cancer. In the present study, the long-term prognosis of VATE was better than that of OS, although no statistically significant difference was observed. The greatest potential benefit of VATE is a reduced incidence of pulmonary complications, which are the most common adverse events following esophageal surgery, with a reported rate as high as $67 \%$ following IS $^{13-15)}$. Tamagawa et al. ${ }^{15)}$ reported that the development of postoperative pneumonia is a risk factor for the OS in patients who undergo esophagectomy for esophageal cancer, and it is necessary to prevent these patients from developing postoperative pneumonia in order to improve their survival. In the present study, the postoperative severe complication rate was lower in the VATE group and respiratory complications in particular were significantly less prevalent in the VATE group than in the OS group. This is thought to be one reason why the prognosis of VATE is better than that of OS. However, the optimal explanation of this discrepancy was unclear. Thus further analysis will focus on this issue.

One of the advantages of VATE for esophageal carcinoma is less blood loss than with the open method ${ }^{7,8)}$. In the present study series, the operation time in the VATE group was significantly longer than that in the OS group, although the blood loss and rate of transfusion were similar between the groups. Furthermore, more lymph nodes tended to have been dissected in the VATE group, and the frequency of recurrent laryngeal nerve injury was higher than in the OS group. Boone et al. ${ }^{16)}$ attributed this finding to the extensive en-bloc lymph node dissection performed in the superior mediastinum. The improved accessibility with VATE allows surgeons to operate further on the deeper side of the thoracic cavity, which cannot be achieved with conventional OS.

Several limitations associated with the present study warrant mention. First, it was a retrospective study with a relatively small sample size, so the results must be confirmed in another cohort or in a prospective multicenter study. Second, there was a time bias in this study, as the data were collected between 2005 and 2018. The surgical procedures, postoperative chemotherapy, and perioperative care might have changed over this period. Furthermore, the test method, detection reagent, and testing time might have differed among studies. Third, we e did not correct the times for thoracic part. Thus, the clinical impact of the thoracic part was unclear. Considering these limitations, the current results should be validated by another study.

In conclusion, the present study showed that VATE is feasible and oncologically safe in select patients and can lead to acceptable surgical results with less postoperative severe or respiratory complications and similar survival outcomes in terms of the long-term outcomes compared with OS.

\section{Consent to publish:}

The study does not involve any personal identifiable data.

\section{Competing interests:}

The authors declare no competing interests in association with the present study

Funding:

None

Acknowledgements:

The work is supported, in part, by the non-governmental organization Yokohama Surgical Research Group, Association of Healthcare corporation, Yoshiki Dermatology Clinic Ginza, and social Hearth Corporation Foundation Pond Friends Association (Fukuoka Wajiro Hospital).

\section{References}

1) Torre, L.A., Bray, F., Siegel, R.L., Ferlay, J., Lortet-Tieulent, J., and Jemal, A. (2015) Global cancer statistics, 2012. CA. Cancer J. Clin., vol. 65: 87-108.

2) Lordick, F., Mariette, C., Haustermans, K., Obermannová, R., Arnold, D., and ESMO Guidelines Committee. (2016) Oesophageal cancer: ESMO Clinical Practice Guidelines for diagnosis, treatment and follow-up. Ann. Oncol. 27: 50-57.

3) Yuequan, J., Shifeng, C., and Bing, Z. (2010) Prognostic factors and family history for survival of esophageal squamous cell carcinoma patients after surgery. Ann. Thorac. Surg. 90: 908-913.

4) Aoyama, T., Hara, K., Kazama K., Atsumi, Y., Tamagawa H., Tamagawa, A., Machida, D., Komori, K., Maezawa, Y., Kano, K., Hashimoto, I., Oshima, T., Murakawa, M., Numata, M., Yukawa, N., Masuda, M., Rino, Y. (2020) The Short- And Long-term Outcomes of Esophagectomy for Esophageal Cancer in Patients Older Than 75 Years. Anticancer Res. 40: 1087-1093.

5) Sabanathan, S., Mearns, A.J., and Richardson, J. (1992) Endoscopic 
oesophagectomy through a right thoracoscopic approach. J. R. Coll. Surg. Edinb. 37: 284-5.

6) Shah, R.D., and D’Amico, T.A. (2014) Modern impact of video assisted thoracic surgery. J. Thorac. Dis. 6: S631-636.

7) Wang, Y., and Chen, C. (2016) Survival Following VideoAssisted Thoracoscopic vs. Open Esophagectomy for Esophageal Carcinoma. J. BUON. 21: 427-433.

8) Wu, X., He, J., Jiang, H., Song, X., Tang, X., Shen, J., Xu, C. (2013) Fully thoracoscopic versus conventional open resection for esophageal carcinoma: A perioperative comparison. Thorac. cancer. 4: 369-372.

9) Taguchi, S., Osugi, H., Higashino, M., Tokuhara, T., Takada, N., Takemura, M., Lee, S., Kinoshita, H. (2003) Comparison of ThreeField Esophagectomy for Esophageal Cancer Incorporating Open or Thoracoscopic Thoracotomy. Surg. Endosc. 17: 1445-1450.

10) Iguchi, K., Kunisaki, C., Sato, S., Tanaka, Y., Miyamoto, H., Kosaka, T., Sato, K., Akiyama, H., Endo, I., Yukawa, N., Rino, Y., Masuda, M., Yamanaka, T. (2020) Efficacy of Video-assisted Thoracoscopic Esophagectomy for Stage II/III Esophageal Cancer: Analysis Using the Propensity Scoring System. Anticancer Res. 40: 1587-1595.

11) Osugi, H., Takemura, M., Higashino, M., Takada, N., Leee, S., Kinoshita, H. (2003) A Comparison of Video-Assisted Thoracoscopic Oesophagectomy and Radical Lymph Node Dissection for Squamous Cell Cancer of the Oesophagus With Open Operation. Br. J. Surg. 90: 108-113.
12) Hsu, P.K., Huang, C.S., Wu, Y.C., Chou, T.Y., Hsu, W.H. (2014) Open vs. Thoracoscopic Esophagectomy in Patients With Esophageal Squamous Cell Carcinoma. World J. Surg. 38: 402409.

13) Bailey, S.H., Bull, D.A., Harpole, D.H., Lentz, J.J., Neumayer, L.A., Pappas, T.N., Daley J., Henderson, W.G., Krasnicka, B., Khuri, S. (2003) Outcomes after esophagectomy: a ten-year prospective cohort. Ann. Thorac. Surg. 75: 217-222.

14) Schieman, C., Wingle, D.A., Deschamps, C., Nichols, I., Cassivi, S.D. (2012) Patterns of operative mortality following esophagectomy. Dis. esophagus. 25: 645-651.

15) Tamagawa, A., Aoyama, T., Tamagawa, H., Ju, M., Komori, K., Maezawa, Y., Kano, K., Kazama, K., Murakawa, M., Atsumi, Y., Sawazaki, S., Hara, K., Numata, M., Sato, T., Yukawa, N., Masuda, M., Rino, Y. (2019) Influence of Postoperative Pneumonia on Esophageal Cancer Survival and Recurrence. Anticancer Res. 39: 2671-2678.

16) Boone, J., Schipper, M.E.I., Moojen, W.A., Borel Rinkes, I.H.M., Cromheecke, G.J.E., von Hillesgersberg, R. (2009) Robot-assisted Thoracoscopic Oesophagectomy for Cancer, Br. J. Surg. 96: 878886. 